\title{
Role of Irisin in Endocrine and Metabolic Disorders-Possible New Therapeutic Agent?
}

\author{
Letitia Leustean ${ }^{1}$, Cristina Preda ${ }^{1}$, Laura Teodoriu ${ }^{1, *}$, Laura Mihalache ${ }^{2}$, Lidia Arhire ${ }^{2} \mathbb{0}$ \\ and Maria-Christina Ungureanu ${ }^{1}$ \\ 1 Endocrinology Department, "Grigore T. Popa" University of Medicine and Pharmacy Iasi, \\ 700111 Iasi, Romania; letitialeustean@yahoo.com (L.L.); cpreda1@yahoo.com (C.P.); \\ mariachristina1105@gmail.com (M.-C.U.) \\ 2 Diabetes and Metabolic Disorders Department, “Grigore T. Popa” University of Medicine and Pharmacy Iasi, \\ 700111 Iasi, Romania; laura_mvlad@yahoo.com (L.M.); lidia_graur@yahoo.com (L.A.) \\ * Correspondence: laurateodoriu@gmail.com
}

Citation: Leustean, L.; Preda, C.; Teodoriu, L.; Mihalache, L.; Arhire, L.; Ungureanu, M.-C. Role of Irisin in Endocrine and Metabolic Disorders-Possible New Therapeutic Agent? Appl. Sci. 2021, 11, 5579. https://doi.org/10.3390/ app11125579

Academic Editor: Veronica Mocanu

Received: 30 April 2021

Accepted: 15 June 2021

Published: 16 June 2021

Publisher's Note: MDPI stays neutral with regard to jurisdictional claims in published maps and institutional affiliations.

Copyright: (c) 2021 by the authors. Licensee MDPI, Basel, Switzerland. This article is an open access article distributed under the terms and conditions of the Creative Commons Attribution (CC BY) license (https:// creativecommons.org/licenses/by/ $4.0 /)$.

\begin{abstract}
Irisin is a novel hormone that provides a possible solution for the treatment of metabolic disorders. Discovered in 2012 by Boström et al., irisin very quickly became an interesting subject in medical research. Irisin has been found in cerebrospinal fluid, the cerebellum, thyroid, pineal gland, liver, pancreas, testis, spleen, adult stomach, and human fetuses. Regarding the actions of irisin, both in animals and humans, the results are contradictory but interesting. Its capability to influence adipose tissue and glycemic homeostasis may be utilized in order to treat hypothyroidism, polycystic ovary syndrome, Prader-Willi syndrome, and other endocrine and metabolic disorders. Considering its osteogenic potential, irisin might be a therapeutic choice in diseases caused by a sedentary lifestyle. New data indicate that irisin treatment may serve in the treatment of severe acute respiratory syndrome coronavirus 2 (SARSCoV-2) infection. Furthermore, several therapeutic agents, such as insulin, metformin, fenofibrate, exenatide, and melatonin, influence the concentrations of irisin in animal models or in humans. Nutritional factors including polyunsaturated fatty acids may also have an effect on irisin concentrations. While it may be "too good to be true," irisin offers many opportunities for future research that would aim to find its optimal therapeutical role in endocrine and metabolic diseases.
\end{abstract}

Keywords: irisin; thyroid; ovaries; osteoporosis; SARSCoV-2

\section{Introduction}

Irisin was discovered by Boström et al. in 2012. During physical exercise, the expression of fibronectin type III domain-containing protein 5 (FDNC5) encoded by FNDC5 gene, increases and undergoes cleavage, resulting in irisin secretion. It is possible that the positive effects of exercise could be mediated by this hormone [1-5].

In recent years, research has brought to attention a multitude of metabolic and nonmetabolic effects of irisin; for example, it has been shown to improve muscle-bone connectivity [6], the browning of white adipose tissue (WAT) [7], and the reduction in insulin resistance and body mass index (BMI) [8-12].

Despite available treatments, there are serious questions related to various diseases which are yet to be answered. Obesity and diabetes, which affect millions of people, as well as polycystic ovary syndrome (PCOS), metabolic syndrome, cardiovascular disease, osteoporosis, and hypothalamic disorders, do not yet benefit from curative treatment.

Taking into account the existing data about the actions of irisin, could this myokine become a novel therapy for all of these diseases? The capability of irisin to regulate adipose tissue, glycemic homeostasis, and the musculoskeletal system may be used in clinical practice in order to manage important chronic morbidities. 
The negative association with BMI and percentage of fat mass might suggest an important role of irisin in the control of obesity [8-12]. Furthermore, the metabolic syndrome and some PCOS phenotypes can be considered as pathologies in which irisin might represent a therapeutic option. Even if the results are somewhat contradictory, regarding the role of irisin in regulating fat tissue and BMI, research in progress is expected to improve upon current knowledge.

Thyroid dysfunctions and hypothalamic disorders may also benefit from the action of irisin, due to its important involvement in weight control. Osteoporosis and sarcopenia could also become new targets for the therapeutic use of irisin [6].

In this narrative review, we present the profile of irisin in several endocrine and metabolic disorders, along with its potential pharmacological uses.

\section{General Data}

Irisin is an approximately $12 \mathrm{kDa}$ hormone-like polypeptide which is produced by proteolytic cleavage of its precursor, FNDC5. The crystal structure of irisin comprises an $\mathrm{N}$-terminal fibronectin 3 (FNIII)-like domain (residues 30-123) and a flexible C-terminal tail (residues 124-140) [13]. Irisine is a myokine, which is secreted following physical activity. Even though skeletal muscle is the source of $72 \%$ of the total amount of circulating irisin, several studies have also suggested other sources, such as pancreatic islets or white adipocyte cells [14]. Recent studies have indicated that irisin acts mostly through mitogen-activated protein kinase (MAPK) signaling pathways, which are involved in neural differentiation, browning of white adipocytes, and osteoblast proliferation, among others. Other essential functions of irisin are mediated through additional pathways, including the $5^{\prime}$ adenosine monophosphate-activated protein kinase (AMPK), phosphatidylinositol 3-kinase/protein kinase B (PI3K/AKT), and signal transducer and activator of transcription 3 (STAT3)/Snail pathways [15]. Irisin may exert its effects through $\alpha \mathrm{V}$ integrin receptors [14].

Apart from the muscle and adipose tissue, irisin is also well-distributed in other organs and tissues, including the cerebrospinal fluid, cerebellum, thyroid, pineal gland, liver, pancreas, testis, spleen, and stomach of adults and fetuses [1].

Regarding the actions of irisin, numerous studies in animal (mice and rat) models and humans have been carried out, with similar or contradictory results.

The main actions of irisin, at the level of the brain, bone, muscle, adipose tissue, liver, pancreas, and heart, are presented in Figure 1.

Irisin acts at the central nervous system level by increasing the multiplication of hippocampal neurons [16] and the expression of brain-derived neurotrophic factor (BDNF); it also plays an important role in neurogenesis and controls feeding behavior through the regulation of peptides/neurotransmitters $[1,17,18]$.

In the skeletal muscle, irisin induces mitochondrial biogenesis [19], stimulates myogenesis by amplifying positive regulators in skeletal muscles [20], and acts as a protection factor against bone loss and muscle atrophy [1].

Cardiovascular and endothelial functions are influenced by irisin, in terms of vasorelaxation and decreased oxidative stress [1,21,22].

The most important and promising actions of irisin involve glucose and lipid homeostasis. The reduction in hepatic insulin resistance, weight and blood glucose levels, inhibition of gluconeogenesis, and stimulation of glycogenesis are the main metabolic effects attributable to irisin $[1,23,24]$. 


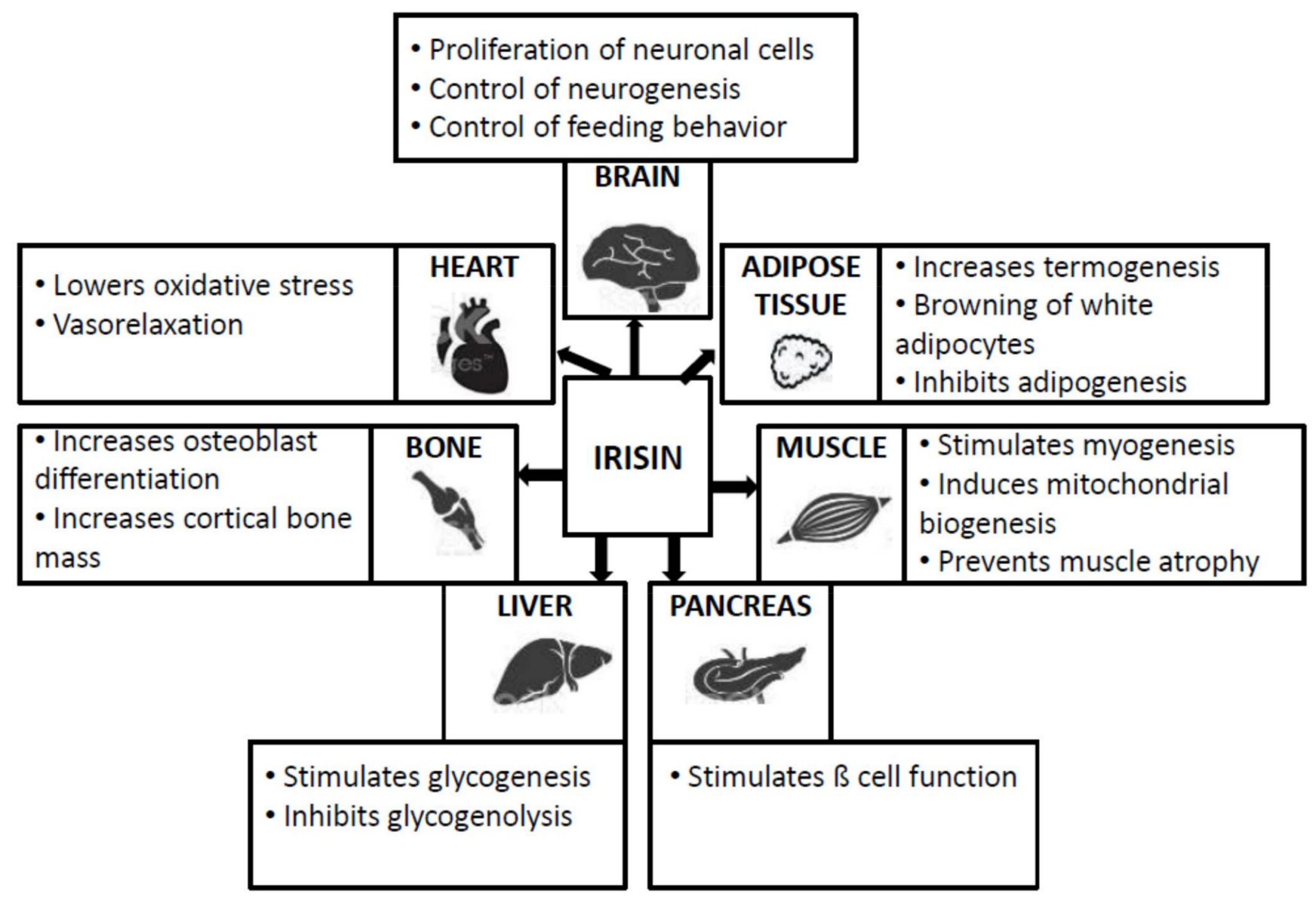

Figure 1. Existing data about the actions of irisin.

There is some debate regarding the measurement of irisin, with uncertain "normal" values of irisin in humans as a consequence. The currently available irisin antibodies still bind to unspecific serum proteins, thus limiting irisin measurement by ELISA. The measurement of irisin with labeled peptides by mass spectrometry is a more accurate method, but requires a complex methodology [25]. Finding a reliable method for irisin determination is mandatory for future studies of this protein, both in humans and experimental animals.

\section{Thyroid Dysfunction}

Current data regarding the connection between serum irisin variations and thyroid function are limited. There is a wide resemblance between the functions of irisin and thyroid hormones, in terms of the homeostatic control of energy balance. The potential impacts of thyroid hormones and thyroid-stimulating hormone (TSH) on irisin levels should be considered [26].

Variations in the serum concentrations of irisin have been observed in several thyroid pathological circumstances characterized by chronic inflammation and hypothyroidism. In primary thyroid insufficiency, an increased level of TSH induces adipogenesis, while hormones such as leptin, ghrelin, and irisin are secreted, in order to balance the fat distribution. Therefore, irisin levels have been found to be high in hypothyroidism, according to the TSH levels. Furthermore, the release of the irisin precursor FNDC5, which is found in the thyroid tissue, into the blood stream provides another explanation for the high level of irisin associated with thyroid chronic inflammation $[27,28]$.

However, irisin values have been shown to negatively correlate with TSH concentrations and positively with fT4 levels in clinical trials enrolling a limited number of patients with thyroid dysfunctions. Hence, irisin concentrations may vary, according to the profile 
of thyroid function [29]. Other non-inflammatory causes of hypothyroidism, as well the limited number of patients, may explain these differences.

Serum irisin levels of males are higher, compared to females, and irisin levels decrease with age [30]. Irisin levels seem to vary with the duration of hypothyroidism: patients with long-lasting hypothyroidism have lower irisin levels than those with recent thyroid insufficiency [26].

In experimental studies, short-term drug-induced hyper- and hypothyroidism were not consistent with previous clinical studies: the up-regulation of serum irisin in male rats with hyper- or hypothyroidism was associated with oxidative damage and/or myopathy observed in both conditions [31].

\section{Small for Gestational Age (SGA)}

SGA children are a well-known group at risk of developing metabolic syndromes and obesity. This condition has been linked to dyslipidemia, hypertension, and hyperglycemia, which are cardio-metabolic risk factors. These complications often carry on into adulthood, resulting in increased morbidity and mortality. The association between SGA infants who have lower muscle mass and brown adipose tissue and decreased glucose tolerance still remains unsolved. Due to the fact that irisin is considered a potential biomarker for metabolic syndrome and overweightness/obesity, one may speculate on the possible role of this myokine in the pathophysiological profile of SGA individuals [32].

In a cross-sectional study of 34 appropriate gestational age (AGA)- and 34 SGA-term newborns, the cord blood irisin levels were found to be decreased in SGA compared to AGA infants, and was positively correlated with birth weight in term infants [32].

A positive correlation between plasma irisin, gestational age, and birth weight was found by Joung et al. [32], providing another argument in the favor of the role of irisin as a pathophysiological factor in impaired carbohydrate metabolism, which is more frequently encountered in SGA infants [32].

\section{Diabetes Mellitus Type 2}

Current research indicates that irisin stimulates the transformation of white adipocytes to brown adipocytes and increases thermogenesis, thus improving glucose tolerance, insulin sensitivity, body weight, and body composition in mice. Studies have shown that irisin promotes glucose uptake in skeletal muscle while improving hepatic glucose and lipid metabolism [11]. Irisin may play an important role in a variety of disorders, including obesity, insulin sensitivity, and diabetes mellitus type 2 (T2DM) [9].

The role of irisin in T2DM remains unclear. Some studies have reported decreased circulating irisin levels in T2DM patients, compared to healthy individuals, whereas studies performed in obese individuals (some of whom had T2DM) have reported elevated levels $[8,10]$. Significantly lower circulating irisin levels have been found in gestational diabetes [12].

In obese T2DM patients, the elevation of circulating irisin may represent a compensatory response to decreased energy expenditure. It is also plausible that excessive adipose tissue in these patients could provide an extra source of irisin. Recent studies in obese subjects have shown that plasma irisin decreases markedly in response to a hypocaloric diet and weight loss, together with significant improvements in hyperglycemia and hyperinsulinemia [10].

It is possible that irisin plays an important role in T2DM-relevant organs, such as the liver and pancreas, but the proposed mechanism(s) of action by which irisin regulates hepatic metabolism and islet function are yet to be solved [11].

\section{Osteoporosis}

Irisin displays anabolic actions on the skeleton. In athletes, the irisin level has been positively correlated with bone mineral density (BMD) and bone strength. Compared to adipose tissue, bone tissue is more sensitive to the action of irisin, where the effect of 
irisin depends on the administered dose. Recombinant irisin (r-irisin), given at a low dose $(100 \mu \mathrm{g} \mathrm{kg} /$ week) in young mice increased cortical bone mineral density and positively modified bone geometry [2].

Irisin exerts its effect on osteoblasts by enhancing the differentiation and activity of bone-forming cells, through increasing activity of transcription factor 4 expression. Lowdose r-irisin also increased osteopontin and decreased sclerostin synthesis. These findings offer an explanation for the positive outcome on the skeleton by skeletal muscle during physical activity [33].

In cultured osteoblasts, irisin enhanced proliferation, differentiation, alkaline phosphatase activity, and mineralization through the activation of p38 mitogen-activated protein kinase and extracellular signal-regulated kinase [34]. Irisin also suppressed osteoclast formation through the inhibition of receptor activator of NF-kB ligand (RANKL)/nuclear factor of activated T-cells, cytoplasmic 1 (NFATc1) in RAW264.7 cells [7].

Significant increases in cortical bone mass and strength have been observed after the administration of r-irisin in mice; in particular, in cortical mineral density and periosteal circumference. This anabolic action was possible through the stimulation of bone formation and reduction in osteoclast number. The trabecular compartments of the same bones were spared [33].

Except for the direct effect of irisin on osteoblasts [34,35], this myokine can also downregulate the expression of the parathormone (PTH) receptor in osteoblast. In a recent study, an inverse association between PTH and irisin concentration was observed after 12 weeks of orthodox fasting among overweight metabolic healthy individuals, indicating a reverse musculoskeletal biological interaction. It could be postulated that additional parameters-including dietary ones-might be implicated in the association between irisin and bone metabolism [36].

Irisin might be a useful biomarker for the assessment of muscle and bone health, due to the fact that, in humans, its synthesis is increased by physical activity [7].

Due to the fact that irisin is diminished in patients with previous osteoporotic fractures and in post-menopausal women [37], it has been speculated that irisin plays a protective role in bone health. It is not known whether this association is independent or whether it is due to low muscle mass [38].

\section{Prader-Willi Syndrome (PW)}

Irisin has both peripheral and central functions, and is suspected to be a molecular link between muscles and the brain. A neural pathway participating in the signal transduction of irisin from the cerebellum to adipocytes through intermediary synapses in the medulla and spinal cord might be involved. Irisin also influences hippocampal neurogenesis (i.e., high concentrations of irisin $50-100 \mathrm{nmol} / \mathrm{L}$ increase neuronal proliferation) [2].

In the hypothalamus, irisin appears to be localized together with neuropeptide Y (NPY) in the neuronal cells of the paraventricular nucleus, while FNDC5mRNA is expressed in the neuroendocrine reproductive axis. Hypothalamic FNDC5 expression is not dependent on nutrition but increases after leptin and insulin infusion [39].

Patients with PW have a specific metabolic profile, compared with BMI-matched controls: insulin sensitivity is increased, and fasting insulin levels are lower. Furthermore, resting energy expenditure is markedly decreased as a consequence of decreased physical activity and decreased lean body mass. In individuals with PW, the visceral adipose tissue is reduced, compared with obese controls [39]. These metabolic particularities of PW support the study of irisin in such patients.

The first study on irisin in PW adults showed increased salivary insulin levels, although plasma irisin was similar to control volunteers. Furthermore, plasma irisin was correlated with salivary insulin in normal controls, but not in PW patients. Salivary irisin significantly correlated with plasma lipids, negatively with HDL cholesterol, and positively with LDL cholesterol and triglycerides. These data suggest that irisin might play a com- 
pensatory role in this hypothalamic syndrome, in order to improve total cholesterol, LDL cholesterol, and HDL cholesterol serum concentrations in obese individuals with PW.

Irisin deregulation may be an answer for the nutritional phase in children with PW aged between 2 and 4 years. In this nutritional phase, they excessively gain weight without an increase in appetite or caloric intake. In contrast, food craving decreased along with irisin normalization in adults with PW [40].

\section{Polycystic Ovary Syndrome}

According to the anticipated function of irisin in reducing insulin resistance, the study of its role in the pathogenesis of PCOS is very promising. PCOS represents a complex endocrine disorder affecting $6-10 \%$ of reproductive women. The clinical manifestations are heterogenous with possible severe metabolic complications; nevertheless, the pathogenesis of PCOS has not yet been fully elucidated.

Peroxisome proliferator-activated receptor gamma coactivator 1-alpha (PGC1 $\alpha)$, which is a transcription factor upstream of FNDC5, plays an important role in ovarian function regulating cellular metabolism and the production of sex hormones. Assessment of the expression of these genes in granulosa cells of a PCOS mouse model showed that the expression of FNDC5 was significantly reduced in granulosa cells of PCOS mice, compared with the control group, while there were no significant differences in PGC1 $\alpha$ expression among different groups. The authors concluded that the downregulation of FNDC5 transcript level may contribute to the metabolic disturbance of granulosa cells derived from ovaries suffering from PCOS, apart from PGC1 $\alpha$ levels, which remained unchanged [41].

Several studies have indicated elevated insulin in PCOS women and have also related it to insulin sensitivity [42]. The mean serum concentrations of irisin before and after insulin infusion are higher in PCOS women, in comparison to the control group. Insulin infusion resulted in a decrease in serum irisin concentration only in the PCOS group. These results lead to the following question: is there an "irisin resistance" in PCOS women similar to "insulin resistance" or "leptin resistance"? [42].

It is possible that increased irisin levels predict insulin resistance, metabolic syndromes, and hyperandrogenemia [43]. Follicular fluid irisin has been correlated with serum irisin in PCOS women. In contrast, there was no significant correlation between serum and follicular fluid irisin levels in poor responders undergoing in vitro fertilization/intracytoplasmic sperm injection (IVF/ICSI) [44]. The elevated serum irisin level can be considered as a protection factor in the pre-diabetic state of PCOS (i.e., before diabetes mellitus develops) [45].

All these data support the assumption that metabolic imbalance triggers a "vicious circle" in PCOS women. As such, the relationship between the adipose tissue, hyperinsulinemia, and hyperandrogenism in PCOS may include a new player; namely, irisin.

\section{Obesity}

Irisin serves as a mediator for the beneficial metabolic effects of physical exercises, by regulating thermogenesis and inhibiting adipogenesis in the adipose tissue [2].

The data regarding the relationship between irisin and adiposity are conflicting, with some authors reporting positive outcomes and others showing negative correlations [2].

Irisin concentrations in obesity are influenced by caloric restriction diets, with high levels of irisin after early intervention. This fact may be associated with greater reductions in blood glucose levels and insulin concentrations. Irisin also increases energy expenditure and, as a result, high levels of triglycerides start providing energy substrates for the organism. When the irisin levels are increased by metabolic abnormalities, weight loss leads to low levels of irisin; thus, the result will be an improved metabolic profile [38].

There are doubts whether higher or lower FNDC5/irisin levels are related to metabolic homeostasis - mainly in patients with metabolic risk-due to excessive body adipose tissue. The results of a cluster study have shown that obese middle-aged men with higher irisin levels exhibit a better metabolic profile, along with a lower risk of T2DM development. 
Some anthropometric/body composition variables, as well as the risk of T2DM, have been inversely related to FNDC5/irisin levels [46].

In obese children, the levels of irisin are higher than in underweight and normal weight children. Irisin has also been positively correlated with adiposity markers (BMI) and negatively correlated with body muscle mass and physical activity. There is no proof of any association between irisin and cardio-metabolic markers in children [47].

In obese patients, irisin has been significantly correlated with the Homeostatic Model Assessment of Insulin Resistance (HOMA-IR) index. Subjects with initial high levels of irisin showed significant improvements in body composition, lipid profile, and fasting glucose after weight loss. These findings suggest that increased irisin exerts beneficial regulatory effects on fat, glycemic control, and insulin resistance [48]. Due to the fact that body weight reduction and exercise influence the kinetics of irisin [48], it may represent a new therapeutic approach for the treatment of obesity [48].

The irisin profile in several endocrine and metabolic disorders are summarized in Table 1.

Table 1. Irisin profiles with respect to various endocrine and metabolic disorders.

\begin{tabular}{|c|c|c|}
\hline Endocrine and Metabolic Disorders & Irisin Profile & References \\
\hline $\begin{array}{l}\text { THYROID } \\
\text { DYSFUNCTIONS }\end{array}$ & $\begin{array}{l}\text { Variation according to the thyroid } \\
\text { function (hypothyroidism or } \\
\text { hyperthyroidism) } \\
\text { Correlation with TSH and fT4 }\end{array}$ & $\begin{array}{l}\text { A. Zybek-Kocik et al., } 2016 \text { [26] } \\
\text { J. Y. Huh et al., } 2012 \text { [27] } \\
\text { M. Ruchala et al., 2014 [29] }\end{array}$ \\
\hline SGA & $\begin{array}{l}\text { Decreased in SGA } \\
\text { Positive correlation with gestational age } \\
\text { and birth weight }\end{array}$ & E. Keles et al., 2016 [32] \\
\hline PRADER-WILLI SD. & $\begin{array}{c}\text { Increased in saliva } \\
\text { Compensatory role for the amelioration } \\
\text { of lipid profile }\end{array}$ & H. J. Hirsh et al., 2015 [40] \\
\hline OSTEOPOROSIS & $\begin{array}{l}\text { Positive correlation with BMD and } \\
\text { bone strength }\end{array}$ & $\begin{array}{l}\text { B. Grygiel-Goriak et al., } 2017 \text { [2] } \\
\text { G. Colaianni et al., } 2014 \text { [33] } \\
\text { X. Qiao et al., 2016 [35] }\end{array}$ \\
\hline OBESITY & $\begin{array}{c}\text { Negative/positive (?) correlation } \\
\text { with adiposity } \\
\text { Positive correlation with BMI in children }\end{array}$ & $\begin{array}{l}\text { B. Grygiel-Goriak et al., } 2017 \text { [2] } \\
\text { L. Elizondo-Montemayor et al., } 2017 \text { [47] }\end{array}$ \\
\hline PCOS & $\begin{array}{l}\text { Increased in serum } \\
\text { Irisin resistance (?) }\end{array}$ & $\begin{array}{l}\text { A. Adamska et al., } 2017 \text { [42] } \\
\text { K. Polak et al., } 2017 \text { [43] } \\
\text { M. Acet et al., } 2016 \text { [44] }\end{array}$ \\
\hline DIABETES MELLITUS & $\begin{array}{c}\text { Decreased in T2DM } \\
\text { Increased in obese T2DM }\end{array}$ & $\begin{array}{l}\text { A. Shoukry et al., } 2016 \text { [8] } \\
\text { K. S. Rana et al., } 2017 \text { [10] } \\
\text { L. Zhao et al., } 2015 \text { [10] }\end{array}$ \\
\hline
\end{tabular}

\section{Irisin: New Therapeutical Agent?}

Irisin supports the relationships between skeletal muscle, adipocytes, bones, and neurons. This specific crosstalk between fat, bone, muscle, and neurons indicates the significant role of this molecule in human metabolic processes [2]. Therefore, irisin can be seen as a possible treatment for diabetes, obesity, Alzheimer's, Parkinson's, and some other neurodegenerative diseases [49].

Considering its metabolic and osteogenic potential, irisin might provide a therapeutic choice for treating diseases caused by sedentary lifestyles. The use of irisin as a new anabolic therapy in osteoporosis, sarcopenia, and tumor-associated cachexia has also shown promise [2]. 
The useful role of irisin in metabolic and osteogenic processes confirms that physical exercise is necessary for the development of the skeleton, the browning process in white adipose tissue, and normal functioning of neurons [2].

However, new studies have started to question the initial expectations. Might the capacity of irisin to promote the "browning" of white adipocytes in mice be transposed to humans? The gene encoding irisin (FNDC5) is expressed robustly not only in muscle but also in various white adipose tissues cells in humans, raising the possibility for increased thermogenesis [3]. No effect of recombinant FNDC5 and irisin on the brightening of primary human adipocytes has been observed, and, thus, the authors concluded that the function of irisin proposed for mice is lost in humans [5,49]. The thermogenic effect of irisin in humans remains controversial, and it is not yet clear whether exercise has an impact on irisin levels.

In human subcutaneous adipocyte cell cultures, irisin had a beneficial effect on the expression of multiple genes related to the SARSCoV-2 viral infection, by causing a reduction in genes implicated in elevated viral infection and an increase in genes that block virus cell cleavage; these results raise hope for the beneficial effects of irisin in the treatment of SARSCoV-2 infection [50].

\section{Irisin and Pharmacological Agents}

Some medications can influence the concentration of irisin. The expression of FNDC5 can also be induced by nutritional factors, including polyunsaturated fatty acids. The supplementation of omega 3 fatty acids significantly induced irisin expression in human rhabdomyosarcoma cells [2].

Recently, statins have been reported to influence the irisin profile, where simvastatin has been shown to increase irisin concentration [2]. Insulin, leptin, metformin, fenofibrate, exenatide, and melatonin have been shown to influence the concentrations of irisin, in animal models or in humans (Table 2).

Table 2. The effects of pharmacological agents on irisin.

\begin{tabular}{|c|c|c|c|}
\hline Therapeutical Agent & Study Type & Action & References \\
\hline INSULIN & humans with T2DM & $\uparrow$ serum irisin & L. Li et al., 2016 [51] \\
\hline LEPTIN & obese mice & $\begin{array}{l}\text { Up-regulation of FNDC5 in } \\
\text { skeletal muscle } \rightarrow \uparrow \text { irisin } \\
\downarrow \text { irisin expression in } \\
\text { subcutaneous fat }\end{array}$ & A. Rodriguez et al., 2015 [52] \\
\hline METFORMIN & diabetic obese mice & $\begin{array}{l}\text { Up-regulation of FNDC5 in } \\
\text { skeletal muscle } \rightarrow \uparrow \text { irisin }\end{array}$ & D. J. Li et al., 2015 [53] \\
\hline METFORMIN & obese mice & $\begin{array}{l}\text { Up-regulation of FNDC5 in } \\
\text { skeletal muscle } \rightarrow \uparrow \text { irisin }\end{array}$ & Z. Yang et al., 2015 [54] \\
\hline FENOFIBRATE & male C57BL/6 mice & irisin induction & T. L. Rachid et al., 2015 [55] \\
\hline FENOFIBRATE & humans with T2DM & $\downarrow$ irisin in serum & X. Feng et al., 2015 [56] \\
\hline SIMVASTATIN & $\begin{array}{l}\text { humans with } \\
\text { hypercholesterolemia }\end{array}$ & $\uparrow$ irisin & I. Gouni-Berthold et al., 2013 [57] \\
\hline EXENATIDE & humans with T2DM & $\uparrow$ irisin & J. Liu et al., 2016 [58] \\
\hline DIHYDROMYRICETIN & $\begin{array}{l}\text { humans } \\
\text { rats }\end{array}$ & $\uparrow$ irisin & Q. Zhou et al., 2015 [59] \\
\hline MELATONIN & Diabetic fatty rats & $\uparrow$ irisin & A. Jimenez-Aranda et al., 2013 [60] \\
\hline
\end{tabular}

\section{Conclusions}

Since the discovery of irisin, much attention has been drawn to its potential therapeutic implications in the treatment of a variety of endocrine and metabolic diseases. 
Taking into account that both irisin and thyroid hormones are associated with metabolism, muscle physiology, and body energy expenditure, as well as the fact that serum irisin concentrations vary, depending on TSH and fT4 in thyroid dysfunction, it can be postulated that irisin and thyroid function are interconnected. The role of irisin in thyroid disorders needs additional research, in terms of animal-based studies, in order to clarify its therapeutic potential in thyroid dysfunctions.

Proving the potential roles of irisin in regulating hepatic and pancreatic islet functions would provide evidence supporting irisin as a mediator for protection against obesity, diabetes, and metabolic syndrome. This will pave the way for irisin to be used as a preventative and therapeutic agent, as well as for PCOS, SGA and hypothalamic syndromic obesity.

The influence of irisin on bone health is still under debate. Clinical (inverse correlation between the number of vertebral menopausal osteoporotic fracture and irisin) and experimental (infusion of r-irisin in mice caused a reduction in osteoclasts and exerted an anabolic effect on the bone) data have suggested that irisin may be an important target for the therapy of decreased bone density. Further studies are necessary, in order to prove irisin's therapeutic potential in osteoporosis.

While it may seem "too good to be true", irisin offers ample opportunities for future research, in order to determine its optimal roles in the treatment of endocrine and metabolic disorders.

Author Contributions: Conceptualization L.L. and C.P.; methodology, M.-C.U.; software, L.T.; validation, L.L., C.P. and M.-C.U.; formal analysis, L.M. and L.A.; investigation, C.P.; resources, L.T.; data curation, L.L.; writing-original draft preparation, L.L.; writing—review and editing, C.P.; visualization, M.-C.U.; supervision, L.M. and L.A. All authors have read and agreed to the published version of the manuscript.

Funding: This research received no external funding.

Institutional Review Board Statement: Not applicable.

Informed Consent Statement: Not applicable.

Data Availability Statement: Not applicable.

Conflicts of Interest: The authors declare no conflict of interest.

\section{References}

1. Mahgoub, M.O.; D'Souza, C.; Al Darmaki, R.S.M.H.; Baniyas, M.M.Y.H.; Adeghate, E. An update on the role of irisin in the regulation of endocrine and metabolic functions. Peptides 2018, 104, 15-23. [CrossRef]

2. Grygiel-Górniak, B.; Puszczewicz, M. A review on irisin, a new protagonist that mediates muscle-adipose-bone-neuron connectivity. Eur. Rev. Med. Pharmacol. Sci. 2017, 21, 4687-4693.

3. Irving, B.A.; Still, C.D.; Argyropoulos, G. Does irisin have a brite future as a therapeutic agent in humans? Curr. Obes. Rep. 2014, 3, 235-241. [CrossRef]

4. Perakakis, N.; Triantafyllou, G.A.; Fernández-Real, J.M.; Huh, J.Y.; Park, K.H.; Seufert, J.; Mantzoros, C.S. Physiology and role of irisin in glucose homeostasis. Nat. Rev. Endocrinol. 2017, 13, 324-337.

5. Raschke, S.; Elsen, M.; Gassenhuber, H.; Sommerfeld, M.; Schwahn, U.; Brockmann, B. Evidence against a beneficial effect of irisin in humans. PLOS ONE 2013, 8, e73680.

6. Colaianni, G.; Grano, M. Role of irisin on the bone-muscle functional unit. Bonekey Rep. 2015, 4, 765.

7. Kaji, H. Effects of myokines on bone. Bonekey Rep. 2016, 5, 826.

8. Shoukry, A.; Shalaby, S.M.; El-ArabiBdeer, S.; Mahmoud, A.A.; Mousa, M.M.; Khalifa, A. Circulating serum irisin levels in obesity and type 2 diabetes mellitus. IUBMB Life 2016, 68, 544-556. [CrossRef] [PubMed]

9. Al-Daghri, N.M.; Mohammed, A.K.; Al-Attas, O.S.; Amer, O.E.; Clerici, M.; Alenad, A.; Alokail, M.S. SNPs in FNDC5 (irisin) are associated with obesity and modulation of glucose and lipid metabolism in Saudi subjects. Lipids Health Dis. 2016, 15, 54. [CrossRef] [PubMed]

10. Rana, K.S.; Pararasa, C.; Afzal, I.; Nagel, D.A.; Hill, E.J.; Bailey, C.J.; Griffiths, H.R.; Kyrou, I.; Randeva, H.S.; Bellary, S.; et al. Plasma irisin is elevated in type 2 diabetes and is associated with increased E-selectin levels. Cardiovasc. Diabetol. $2017,16,147$.

11. Leung, P.S. The potential of irisin as a therapeutic for diabetes. Future Med. Chem. 2017, 9, 529-532. [CrossRef]

12. Zhao, L.; Li, J.; Li, Z.L.; Yang, J.; Li, M.L.; Wang, G.L. Circulating irisin is lower in gestational diabetes mellitus. Endocr. J. 2015, $62,921-926$. 
13. Askari, H.; Rajani, S.F.; Poorebrahim, M.; Haghi-Aminjan, H.; Raeis-Abdollahi, E.; Abdollahi, M. A glance at the therapeutic potential of irisin against diseases involving inflammation, oxidative stress, and apoptosis: An introductory review. Pharmacol. Res. 2018, 129, 44-55. [CrossRef] [PubMed]

14. Marrano, N.; Biondi, G.; Borrelli, A.; Cignarelli, A.; Perrini, S.; Laviola, L.; Giorgino, F.; Natalicchio, A. Irisin and incretin hormones: Similarities, differences, and implications in type 2 diabetes and obesity. Biomolecules 2021, 11, 286. [CrossRef]

15. Rabiee, F.; Lachinani, L.; Ghaedi, S.; Nasr-Esfahani, M.H.; Megraw, T.L.; Ghaedi, K. New insights into the cellular activities of Fndc5/irisin and its signaling pathways. Cell Biosci. 2020, 10, 51. [CrossRef] [PubMed]

16. Moon, H.S.; Dincer, F.; Mantzoros, C.S. Pharmacological concentrations of irisin increase cell proliferation without influencing markers of neurite outgrowth and synaptogenesis in mouse H19-7 hippocampal cell lines. Metabolism 2013, 62, 1131-1136. [CrossRef]

17. Tekin, S.; Erden, Y.; Ozyalin, F.; Cigremis, Y.; Colak, C.; Sandal, S. The effects of intracerebroventricular infusion of irisin on feeding behaviour in rats. Neurosci. Lett. 2017, 645, 25-32. [CrossRef]

18. Wrann, C.D. FNDC5/irisin-their role in the nervous system and as a mediator for beneficial effects of exercise on the brain. Brain Plast. 2015, 1, 55-61. [CrossRef] [PubMed]

19. Vaughan, R.A.; Gannon, N.P.; Barberena, M.A.; Garcia-Smith, R.; Bisoffi, M.; Mermier, C.M.; Conn, C.A.; Trujillo, K.A. Characterization of the metabolic effects of irisin on skeletal muscle in vitro. Diabetes Obes. Metab. 2014, 16, 711-718.

20. Rodriguez, A.; Becerril, S.; Mendez-Gimenez, L.; Ramirez, B.; Sainz, N.; Catalan, V.; Gómez-Ambrosi, J.; Frühbeck, G. Leptin administration activates irisin-induced myogenesis via nitric oxidedependent mechanisms, but reduces its effect on subcutaneous fat browning in mice. Int. J. Obes. 2015, 39, 397-407. [CrossRef]

21. Jiang, M.; Wan, F.; Wang, F.; Wu, Q. Irisin relaxes mouse mesenteric arteries through endothelium-dependent and endotheliumindependent mechanisms. Biochem. Biophys. Res. Commun. 2015, 468, 832-836. [CrossRef] [PubMed]

22. Han, F.; Zhang, S.; Hou, N.; Wang, D.; Sun, X. Irisin improves endothelial function in obese mice through the AMPK-eNOS pathway. Am. J. Physiol. Heart Circ. Physiol. 2015, 309, 1501-1508. [CrossRef]

23. Xiong, X.Q.; Chen, D.; Sun, H.J.; Ding, L.; Wang, J.J.; Chen, Q.; Li, Y.H.; Zhou, Y.B.; Han, Y.; Zhang, F.; et al. FNDC5 overexpression and irisin ameliorate glucose/lipid metabolic derangements and enhance lipolysis in obesity. Biochim. Biophys. Acta 2015, 1852, 1867-1875. [CrossRef] [PubMed]

24. So, W.Y.; Leung, P.S. Irisin ameliorates hepatic glucose/lipid metabolism and enhances cell survival in insulin-resistant human HepG2 cells through adenosine monophosphate-activated protein kinase signaling. Int. J. Biochem. Cell. Biol. 2016, 78, 237-247. [CrossRef]

25. Albrecht, E.; Schering, L.; Buck, F.; Vlach, K.; Schober, H.C.; Drevon, C.A.; Maak, S. Irisin: Still chasing shadows. Mol. Metab. 2020, 34, 124-135.

26. Zybek-Kocik, A.; Sawicka-Gutaj, N.; Wrotkowska, E.; Sowiński, J.; Ruchała, M. Time-dependent irisin concentration changes in patients affected by overt hypothyroidism. Endokrynol. Pol. 2016, 67, 476-480.

27. Huh, J.Y.; Panagiotou, G.; Mougios, V.; Brinkoetter, M.; Vamvini, M.T.; Schneider, B.E.; Mantzoros, C.S. FNDC5 and irisin in humans: I. predictors of circulating concentrations in serum and plasma and II. mRNA expression and circulating concentrations in response to weight loss and exercise. Metabolism 2012, 61, 1725-1738. [CrossRef] [PubMed]

28. Dua, A.; Hennes, M.I.; Hoffmann, R.G.; Maas, D.L.; Krakower, G.R.; Sonnenberg, G.E.; Kissebah, A.H. Leptin: A significant indicator of total body fat but not of visceral fat and insulin insensitivity in African-American women. Diabetes 1996, 45, 1635-1637. [CrossRef] [PubMed]

29. Ruchala, M.; Zybek, A.; Szczepanek-Parulska, E. Serum irisin levels and thyroid function-newly discovered association. Peptides 2014, 60, 51-55. [CrossRef]

30. Ateş, İ.; Altay, M.; Topçuoğlu, C.; Yılmaz, F.M. Circulating levels of irisin is elevated in hypothyroidism, a case-control study. Arch. Endocrinol. Metab. 2016, 60, 95-100. [CrossRef]

31. Samy, D.M.; Ismail, C.A.; Nassra, R.A. Circulating irisin concentrations in rat models of thyroid dysfunction-effect of exercise. Metabolism 2015, 64, 804-813. [CrossRef] [PubMed]

32. Keleş, E.; Turan, F.F. Evaluation of cord blood irisin levels in term newborns with small gestational age and appropriate gestational age. Springerplus 2016, 5, 1757. [CrossRef] [PubMed]

33. Colaianni, G.; Cuscito, C.; Mongelli, T.; Oranger, A.; Mori, G.; Brunetti, G.; Colucci, S.; Cinti, S.; Grano, M. Irisin enhances osteoblast differentiation in vitro. Int. J. Endocrinol. 2014, 2014, 902186. [CrossRef] [PubMed]

34. Chen, Z.; Zhang, Y.; Zhao, F.; Yin, C.; Yang, C.; Wang, X.; Wu, Z.; Liang, S.; Li, D.; Lin, X.; et al. Recombinant irisin prevents the reduction of osteoblast differentiation induced by stimulated microgravity through increasing $\beta$-catenin expression. Int. J. Mol. Sci. 2020, 21, 1259. [CrossRef]

35. Qiao, X.; Nie, Y.; Ma, Y.; Chen, Y.; Cheng, R.; Yin, W.; Hu, Y.; Xu, W.; Xu, L. Irisin promotes osteoblast proliferation and differentiation via activating the MAP kinase signaling pathways. Sci. Rep. 2016, 6, 18732. [CrossRef]

36. Karras, S.N.; Koufakis, T.; Adamidou, L.; Dimakopoulos, G.; Karalazou, P.; Thisiadou, K.; Makedou, K.; Kotsa, K. Effects of christian orthodox fasting versus time-restricted eating on plasma irisin concentrations among overweight metabolically healthy individuals. Nutrients 2021, 13, 1071. [CrossRef] [PubMed]

37. Engin-Üstün, Y.; Çağlayan, E.K.; Göçmen, A.Y.; Polat, M.F. Postmenopausal osteoporosis is associated with serum chemerin and irisin but not with apolipoprotein M levels. J. Menopausal Med. 2016, 22, 76-79. [CrossRef] [PubMed] 
38. Gouveia, M.C.; Vella, J.P.; Cafeo, F.R.; Affonso Fonseca, F.L.; Bacci, M.R. Association between irisin and major chronic diseases: A review. Eur. Rev. Med. Pharmacol. Sci. 2016, 20, 4072-4077.

39. Varela-Rodríguez, B.M.; Pena-Bello, L.; Juiz-Valiña, P.; Vidal-Bretal, B.; Cordido, F.; Sangiao-Alvarellos, S. FNDC5 expression and circulating irisin levels are modified by diet and hormonal conditions in hypothalamus, adipose tissue and muscle. Sci. Rep. 2016, 6, 29898. [CrossRef] [PubMed]

40. Hirsch, H.J.; Gross, I.; Pollak, Y.; Eldar-Geva, T.; Gross-Tsur, V. Irisin and the metabolic phenotype of adults with prader-willi syndrome. PLoS ONE 2015, 10, e0136864.

41. Bakhshalizadeh, S.; Rabiee, F.; Shirazi, R.; Ghaedi, K.; Amidi, F.; Nasr-Esfahani, M.H. Assessment of PGC1 $\alpha$-FNDC5 axis in granulosa cells of PCOS mouse model. J. Reprod. Infertil. 2018, 19, 89-94.

42. Adamska, A.; Karczewska-Kupczewska, M.; Lebkowska, A.; Milewski, R.; Gorska, M.; Otziomek, E. Serum irisin and its regulation by hyperinsulinemia in women with polycystic ovary syndrome. Endocrine. J. 2017, 63, 1107-1112.

43. Polak, K.; Czyzyk, A.; Simoncini, T.; Meczekalski, B. New markers of insulin resistance in polycystic ovary syndrome. J. Endocrinol. Investig. 2017, 40, 1-8. [CrossRef] [PubMed]

44. Acet, M.; Celik, N.; Acet, T.; Ilhan, S.; Yardim, M.; Aktun, H.L.; Basaranoglu, S.; Deregozu, A.; Aydin, S. Serum and follicular fluid irisin levels in poor and high responder women undergoing IVF/ICSI. Eur. Rev. Med. Pharmacol. Sci. 2016, 20, 1940-1946. [PubMed]

45. Bostancı, M.S.; Akdemir, N.; Cinemre, B.; Cevrioglu, A.S.; Özden, S.; Ünal, O. Serum irisin levels in patients with polycystic ovary syndrome. Eur. Rev. Med. Pharmacol. Sci. 2015, 19, 4462-4468.

46. Bonfante, I.L.; Chacon-Mikahil, M.P.T.; Brunelli, D.T.; Gáspari, A.F.; Duft, R.G.; Oliveira, A.G.; Araujo, T.G.; Saad, M.J.A.; Cavaglieri, C.R. Obese with higher FNDC5/irisin levels have a better metabolic profile, lower lipopolysaccharide levels and type 2 diabetes risk. Arch. Endocrinol. Metab. 2017, 61, 524-533.

47. Elizondo-Montemayor, L.; Silva-Platas, C.; Torres-Quintanilla, A.; Rodríguez-López, C.; Ruiz-Esparza, G.U.; Reyes-Mendoza, E.; Garcia-Rivas, G. Association of irisin plasma levels with anthropometric parameters in children with underweight, normal weight, overweight, and obesity. Biomed. Res. Int. 2017, 2017, 2628968.

48. Fukushima, Y.; Kurose, S.; Shinno, H.; Thi Thu, H.C.; Takao, N.; Tsutsumi, H.; Hasegawa, T.; Nakajima, T.; Kimura, Y. Effects of body weight reduction on serum irisin and metabolic parameters in obese subjects. Diabetes Metab. J. 2016, 40, 386-395. [CrossRef]

49. Novelle, M.G.; Contreras, C.; Romero-Picó, A.; López, M.; Diéguez, C. Irisin, two years later. Int. J. Endocrinol. 2013, $2013,746281$.

50. De Oliveira, M.; De Sibio, M.T.; Mathias, L.S.; Rodrigues, B.M.; Sakalem, M.E.; Nogueira, C.R. Irisin modulates genes associated with severe coronavirus disease (COVID-19) outcome in human subcutaneous adipocytes cell culture. Mol. Cell Endocrinol. 2020, 515, 110917. [CrossRef] [PubMed]

51. Li, L.; Rampersad, S.; Wang, X.; Cheng, X.; Qu, S. Serum irisin concentrations were increased after transient continuous subcutaneous insulin infusion in type 2 diabetes mellitus patients. Diabetes Res. Clin. Pract. 2016, 113, 44-47. [CrossRef] [PubMed]

52. Rodrigues, K.C.D.C.; Pereira, R.M.; de Campos, T.D.P.; De Moura, R.F.; Da Silva, A.S.R.; Cintra, D.E.; Ropelle, E.R.; Pauli, J.R.; de Araújo, M.B.; Moura, L.P. The role of physical exercise to improve the browning of white adipose tissue via POMC neurons. Front. Cell Neurosci. 2018, 12, 88. [CrossRef]

53. Li, D.J.; Huang, F.; Lu, W.J.; Jiang, G.J.; Deng, Y.P.; Shen, F.M. Metformin promotes irisin release from murine skeletal muscle independently of AMP-activated protein kinase activation. Acta Physiol. 2015, 213, 711-721. [CrossRef] [PubMed]

54. Yang, Z.; Chen, X.; Chen, Y.; Zhao, Q. PGC-1 mediates the regulation of metformin in muscle irisin expression and function. Am. J. Transl. Res. 2015, 7, 1850-1859.

55. Rachid, T.L.; Penna-de-Carvalho, A.; Bringhenti, I.; Aguila, M.B.; Mandarim-de Lacerda, C.A.; Souza-Mello, V. Fenofibrate, (PPARalpha agonist) induces beige cell formation in subcutaneous white adipose tissue from diet-induced male obese mice. Mol. Cell. Endocrinol. 2015, 402, 86-94. [CrossRef]

56. Feng, X.; Gao, X.; Jia, Y.; Zhang, H.; Pan, Q.; Yao, Z.; Yang, N.; Liu, J.; Xu, Y.; Wang, G.; et al. PPAR- $\alpha$ agonist fenofibrate decreased serum irisin levels in type 2 diabetes patients with hypertriglyceridemia. PPAR Res. 2015, 2015, 924131.

57. Gouni-Berthold, I.; Berthold, H.K.; Huh, J.Y.; Berman, R.; Spenrath, N.; Krone, W.; Mantzoros, C.S. Effects of lipid-lowering drugs on irisin in human subjects in vivo and in human skeletal muscle cells ex vivo. PLoS ONE 2013, 8, e72858.

58. Liu, J.; Hu, Y.; Zhang, H.; Xu, Y.; Wang, G. Exenatide treatment increases serum irisin levels in patients with obesity and newly diagnosed type 2 diabetes. J. Diabetes Complicat. 2016, 30, 1555-1559.

59. Zhou, Q.; Chen, K.; Liu, P.; Gao, Y.; Zou, D.; Deng, H.; Huang, Y.; Zhang, Q.; Zhu, J.; Mi, M. Dihydromyricetin stimulates irisin secretion partially via the PGC-1 $\alpha$ pathway. Mol. Cell. Endocrinol. 2015, 412, 349-357.

60. Jiménez-Aranda, A.; Fernández-Vázquez, G.; Campos, D.; Tassi, M.; Velasco-Perez, L.; Tan, D.X.; Reiter, R.J.; Agil, A. Melatonin induces browning of inguinal white adipose tissue in Zucker diabetic fatty rats. J. Pineal Res. 2013, 55, 416-423. [CrossRef] [PubMed] 